\title{
GPS-Based Location Estimation System in a NLOS Environment Using a Screen Coordinate System
}

\author{
Min-HoJeon and Chang-heon Oh \\ School of Electrical, Electronics \& Communication Engineering, Korea University of \\ Technology and Education \\ \{w1004me,choh\}@koreatech.ac.kr
}

\begin{abstract}
GPS-based location estimation systems are the optimum way to track where an object is in an outdoor environment. However, location errors occur when signals transmitted by GPS satellites are interfered with by buildings or other obstacles, since the location tracking system uses these signals. To modify the positional errors incurred by GPS signal interference, this study proposes a measure to minimize the errors of GPS-based location estimation systems in NLOS environments. In this system, location errors are reduced by organizing the screen coordinates with collected image data, establishing an environment favorable for the application of visual information to the screen coordinate system. This data is then applied using perspective projection formulas, substituting the actual GPS data to the divided screen. When tracking a person located at the base of a building, the error range was reduced by around5m when using the proposed system as compared to GPS signals alone.
\end{abstract}

Keywords: GPS, location estimation, screen coordinate system, LOS, NLOS

\section{Introduction}

Global positioning system (GPS) information is commonly used to track the location of a person in both indoor and outdoor environments, and a wide range of studies have been performed to track a person's location using GPS information [1, 2].There are currently personal location tracking services which use GPS information and the Google Earth API, and these provide a very precise location generally. However, positional errors occur when tracking a person or an object located in an area with high-rise buildings or narrow roads. In particular, when tracking a person located at the base of a tall building, large errors can occur. To improve this shortcoming, studies have been conducted to provide location information by distinguishing a person or an object using imagery information. However, the use of imagery information alone is problematic as it is unable to provide a standard location or to extract information about a person located in a specific place correctly [3-5]. To resolve these issues, this study proposes a GPS-based location estimation system for a NLOS environment, using a screen coordinate system to provide a standard location and the person's information when signal interference occurs. The proposed system is composed of offline and location estimation phases. In the offline phase, information is gathered to estimate the location. First, perspective coordinates are projected onto two-dimensional images using perspective projection equations to reduce errors occurring whilst dividing collected images and then sections are created by partitioning the coordinates of the images. Next, a system for location estimation is set up by substituting GPS data gathered from Google Earth to the middle of the created sections. In the location estimation phase, the location is estimated using data gathered and established in the offline phase. Motion detected regions are searched first using 
block matching algorithms in the gathered images and GPS data information having similar locations is shifted to the GPS position matched with the screen coordinates by comparing with the collected GPS data.

The results show that errors occurring beside and at the base of tall buildings were significantly reduced when compared to the location estimation systems proposed in this study and those from Google Earth. The error range markedly decreased when sections were further subdivided.

This paper is composed as follows:

Section 2 explains the offline phase, the first phase of the proposed system, and Section 3 states the location estimation phase, where the position is tracked using the system set up in the offline phase. Section 4 evaluates the performance of the proposed system, and Section 5 reports the conclusions.

\section{Phase 1 of the GPS-based Location Estimation System in a NLOS Environment using Screen Coordinates: The Offline Phase}

The idea of the proposed system was first originated from studies performed using the fingerprinting technique. The fingerprinting technique establishes a database by gathering information on the positions to be tracked, and estimates the locations by matching the established database with the collected positional information. However, this technique is mainly used in indoor environments and estimates the positions using RSSI signals received from wireless devices such as WLAN, Bluetooth, and others. For this reason, different variables need to be taken into consideration, such as a person's location, orientation, the structure of the building and the number of people in the vicinity, amongst other factors. When estimating the positions after receiving the GPS information using smartphones in outdoor environments, the fingerprinting technique is a highly favorable method for location estimation. However, a position estimation system using the fingerprinting technique and GPS information is ineffective since errors do not occur in GPS data. Therefore, the authors of this study have examined ways to alter the fingerprinting technique for other environments. The offline phase of the fingerprinting method explained in this section is appropriately modified for the system proposed in this study.

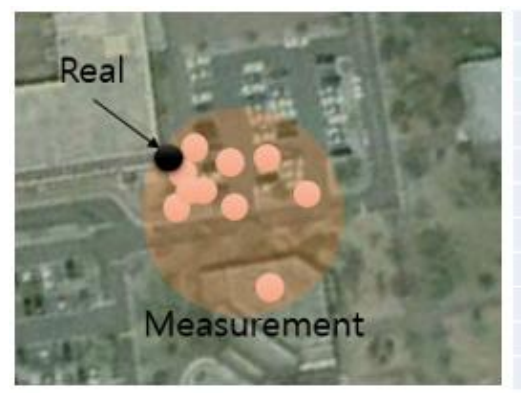

\begin{tabular}{|c|c|c|}
\hline No & Latitude $(\mathrm{N})$ & Hardness $(E)$ \\
\hline 1 & $36^{\prime} 45.877$ & $127^{\prime} 16.817$ \\
\hline 2 & 36.45 .882 & $127^{\prime} 16.798$ \\
\hline 3 & $36^{\prime} 45.881$ & $127^{\prime} 16.798$ \\
\hline 4 & $36^{\prime} 45.891$ & $127^{\prime} 16.805$ \\
\hline 5 & 3645.882 & $127^{`} 16.796$ \\
\hline 6 & $36^{\prime} 45.886$ & $127 \times 16.799$ \\
\hline 7 & 3645.887 & $127^{\prime} 16.809$ \\
\hline 8 & $36^{\prime} 45.884$ & $127^{`} 16.797$ \\
\hline 9 & 36.45 .883 & $127^{\prime} 16.801$ \\
\hline 10 & $36^{\prime} 45.88$ & $127 \times 16.795$ \\
\hline
\end{tabular}

Figure 1.GPS distance errors ina NLOS environment

Firstly, the data necessary to apply the fingerprinting technique is collected at the base of a building to reduce the GPS position errors in a NLOS environment. Figure 1 shows an image of GPS data gathered at the base of a building along with chronological data.The GPS data was received every $30 \mathrm{~min}$ at the same location, however the measured data varied at every instance and it was possible to identify measurement errors between the location indicated by GPS and the actual measurement interval. The outcome indicates that measurement of the 
signal receiving location cannot be repeated using the latitude and path of the database, after establishment of the database using the actual incoming data, and cannot be used since the measured data has its own values in the LOS environment.

To resolve these issues, this research utilized image information. GPS data was first substituted into the image to track the standard position using image information. A small error occurrence rate was taken into account in setting sections, since storing the GPS data for each pixel proved to be complex. Since three-dimensional images of collected information are provided as two-dimensional images, the perspective needs to be projected. To establish the perspective of incoming three-dimensional images, distance is first substituted in order to convert two-dimensional images to three-dimensional images using perspective projection formulas [6,7]. Subsequently, sections are set up in the two-dimensional images and are projected with perspective using equations 1 and 2.In equations 1 and $2, \alpha$ denotes the number of sections, wis the width, and $h$ is the height.

$$
\begin{array}{r}
S_{h i}(\alpha)=h / \alpha \\
S_{w j}(\alpha)=w / \alpha
\end{array}
$$

Figure 2(a) shows an image of sections established by dividing the screen using image information projected with perspective from equations (1) and (2), and Figure 2(b) represents the image of the Google Earth coordinates substituted into each section.

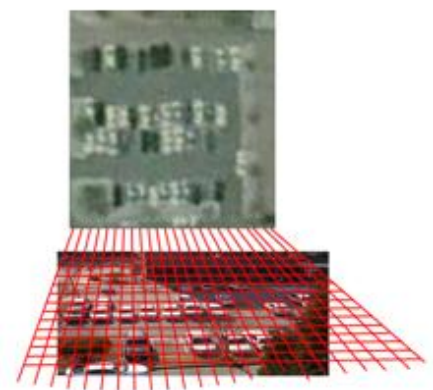

(a)

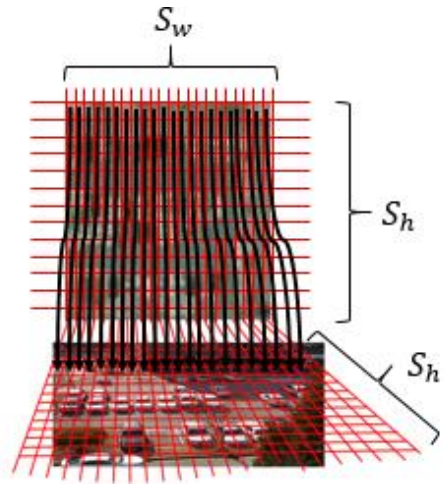

(b)

Figuers 2. Establishment of sectionsand GPS dataprojection

\section{Phase 2 of the GPS-based Location Estimation System in a NLOS Environment using Screen Coordinates: The Location Estimation Phase}

The location estimation phase estimates the positions using the GPS coordinates of image information projected with data gathered from the offline phase and image processing algorithms. This study used block matching algorithms in order to detect motion. The change detection method for the image information uses more than two frames for detection, and extracts the changed parts by comparing each pixel after image information conversion frame by frame to binary image data. Figure 3 shows the different parts detected using block matching algorithms. The changed areas are output as white parts, and the areas with a large number of white parts are determined to be the sections with movement and the presence of 
people. Figure 4 shows the final movement detection for people in images. The proposed system only detectsthe motion of people as a result of the constant position of buildings and other objects [8-10].

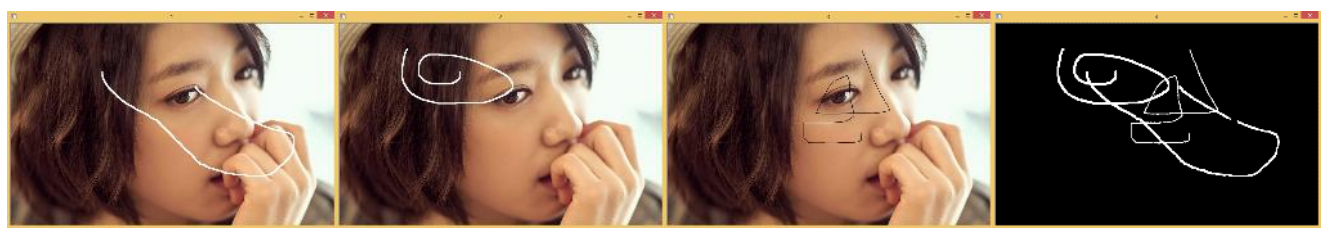

Figure 3. Detection of different parts using block matching algorithms

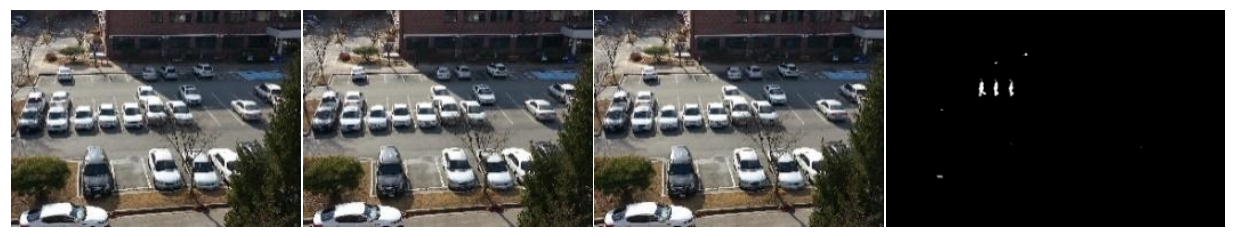

Figure 4. Movement detection forpeople in images

When the movement of people is detected, GPS data transmitted from smartphones and GPS pixels which are white are compared, and the GPS information of the actual location is provided by replacing the image-matched GPS information with the received GPS information.

\section{Implementation and evaluation}

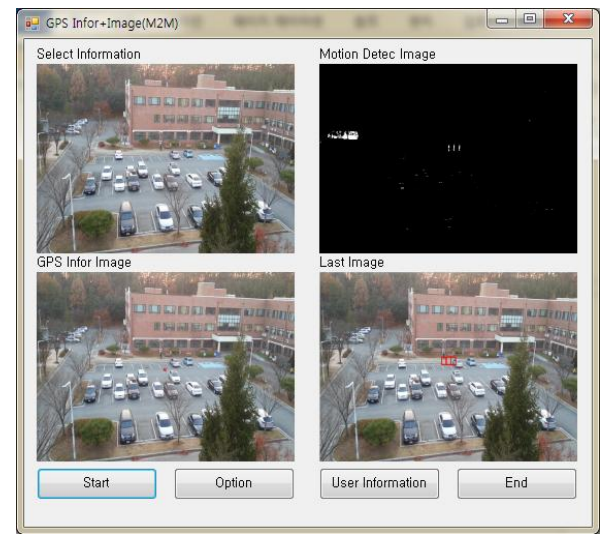

Figure 5. GPS data measurement system using image information

To test the proposed system, a GPS-based location estimation system was established using.Net Framework based C\# language. The GPS coordinates of the image information were set up in the offline phase after installing the image collecting instrument at the location where the positional data was gathered. Figure 5 represents an executable file developed for the tests of this study.

To perform tests for accurately estimating the positions using GPS information in a NLOS environment, GPS data was collected in a NLOS environment and transmitted to a server via Bluetooth. Subsequently, the Google Earth locations were compared by projecting the 
collected GPS information and GPS information modified with the proposed system. Figure 6 shows the results taken when passing through a NLOS area. A comparison of the existing system to the proposed system shows that errors ranged from a minimum of $3 \mathrm{~m}$ to a maximum of $6 \mathrm{~m}$ in the existing system. On the other hand, errors in the proposed system ranged from a minimum of $24 \mathrm{~cm}$ to a maximum of $58 \mathrm{~cm}$.

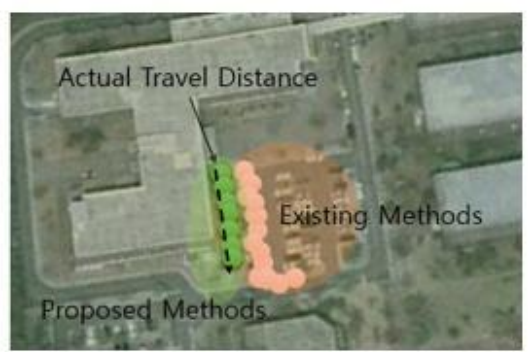

\begin{tabular}{|c|c|c|c|}
\hline \multicolumn{2}{|c|}{ Existing Methods } & \multicolumn{2}{|c|}{ Proposed Methods } \\
\hline 3645.894 & 12716.787 & $36455 \div 3.55$ & $127^{\prime} 16^{\circ} 46.93$ \\
\hline $36 \_45.894$ & $127^{\prime} 16.788$ & $366^{\prime} 5^{\prime} 53.48$ & 1271647.06 \\
\hline $36^{\circ} 45.893$ & 12716.788 & $36 \div 45 \div 53.43$ & $127 \times 16^{\prime} 47.15$ \\
\hline $36 \div 45.893$ & 12716.789 & $36.45^{\prime} 53.28$ & $127^{\prime} 16^{\prime} 47.30$ \\
\hline $36^{\circ} 45,893$ & $127 \times 16.790$ & $36^{\prime} 45^{\prime} 53.20$ & $127^{\prime} 16^{\prime} 47.42$ \\
\hline$3 6 \longdiv { 4 5 . 8 9 3 }$ & $127 \times 16.791$ & $36455^{\circ} 53.15$ & $12716^{\prime} 47.47$ \\
\hline $36^{\circ} 45.893$ & $127^{\prime} 16.792$ & $36.45^{\circ} 53.09$ & $127^{\prime} 16^{\prime} 47.51$ \\
\hline 36.45 .892 & 12716.793 & 364553.05 & $127^{\prime} 16^{\prime} 46.58$ \\
\hline $36 ׳ 45.891$ & $127^{\prime} 16.794$ & $36 ׳ 45 \div 52.98$ & 1271647.64 \\
\hline
\end{tabular}

Figure 6. A comparison of positional errorsusing aGoogle Earth frame

Figure 7 represents the errors against their distances from the building. A decreasing tendency in the error rate was identified in the existing method as distances from the building increased. An error range similar to that of the proposed system was confirmed.

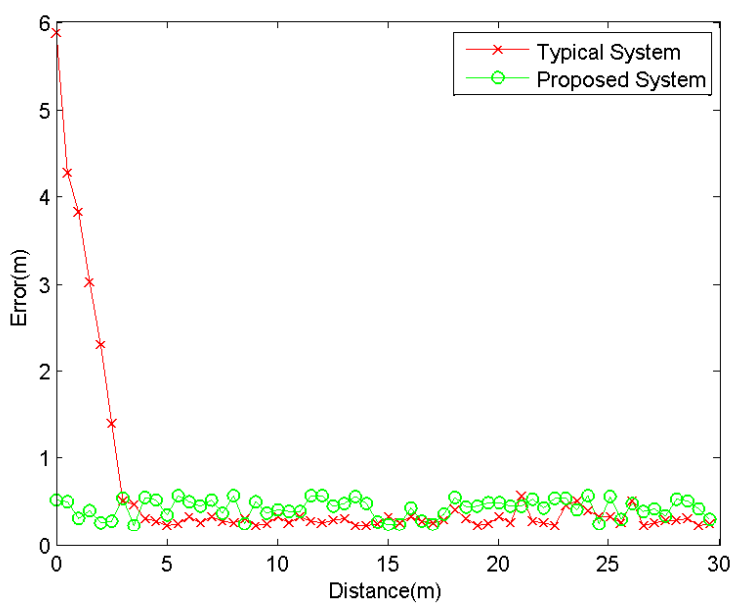

Figure 7. Positional error magnitudeagainstthe distance from the building

\section{Conclusions}

This study proposes a GPS-based location estimation system using a screen coordinates as a measure, to minimize the GPS position errors in a NLOS environment. To modify the positional errors incurred by GPS signal interference, the proposed system organizes screen coordinates with collected image data, and creates an environment favorable for visual information to be applied to the screen coordinate system. This visual information is converted using perspective projection formulas, and the actual GPS data is substituted to the divided screen. The proposed system comprises offline and location estimation phases and minimizes the positional errors of a GPS-based location estimation system in the NLOS environment. This study was able to verify that the error range was reduced by around $5 \mathrm{~m}$ in 
the proposed system compared to the existing location estimation method in a NLOS environment. Moreover, the proposed system exhibited a similar performance to the existing system in a LOS environment. Additional studies will be performed to improve the position tracking system for different environments by further investigating an algorithm that recognizes the border between LOS and NLOS environments.

\section{Acknowledgements}

This research was supported by the MSIP (Ministry of Science, ICT \& Future Planning), Korea, under IT/SW Creative research program supervised by the NIPA(National IT Industry Promotion Agency)" (NIPA-2013-(H0502-13-1082))

\section{References}

[1] K. Virrantaus, J. Markkula, A. Garmash and Y. V. Terziyan, J. IEEE Web information system engineering, vol. 2, no. 66, (2001).

[2] R. Want, A. Hopper, V. Falcao and J. Gibbons, J. ACM Transactions on information systems, vol. 10, no. 91, (1992).

[3] M. Sungryong and S. Seong, Institute of Electronics Engineers of Korea, vol. 3, no. 11, (2010).

[4] K. Changgeun, K. sounggyun and K. hyun-ju, Information and communication convergence Engineering, vol. 11, no. 56, (2013).

[5] L. Woongsup and K. kill, J. Information and communication convergence engineering, vol. 11, no. 167, (2013).

[6] J. D. Foley, A. V. Dam, S. K. Feiner, J. F. Hughes and R. L. Phillips, Editor, "Introduction to Computer Graphics", Addison-Wesley Professional, Chicago, (1993).

[7] J. Minho and O. Changheon, (Eds.), Science and Engineering Research Support Society, Proceedings of the 37th Advanced Science and Technology Letters (ASTL), Jeju Island, Korea (2013) December 11-13.

[8] D. T. Levin and D. J. Simons, J. Psychonomic Bulletin \& Review, vol. 4, no. 501, (1997).

[9] R. Cutler and L. S. Davis, J. Patten Analysis and Machine Intelligence, vol. 22, no. 781, (2000).

[10] A. C. Bovik, Editor, "Al Bovik, working togerther to grow libraries in developing countries", Massachusetts, (2010).

\section{Authors}

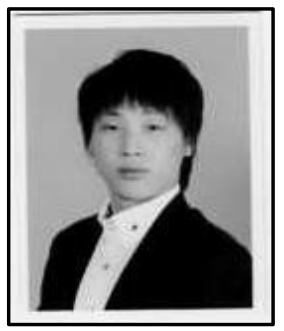

\section{Min-Ho Jeon}

He received aB.S degree in the Department of Game Digital Content from Far East University, Umsung, Korea, in 2009, and an M.S. degree in Electrical, Electronics and Communication Engineering at the Korea University of Technology and Education, Cheonan, Korea, in 2011. He is currently pursuing a Ph.D. degree in Electrical, Electronics and Communication Engineering at the Korea University of Technology and Education, Cheonan, Korea. His research interests are in the context-aware, wireless sensor network, wireless localization, channel coding and M2M network. 


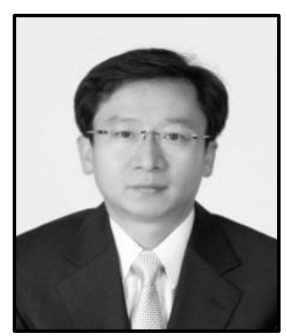

\section{Chang-Heon Oh}

He received the B. S. and M.S.E. degrees in telecommunication and informa tion engineering from Korea Aerospace Univ. in 1988 and 1990, respectively. He received the Ph.D. degree in avionics engineering from Korea Aerospace Univ., in 1996. From Feb. 1990 to Aug. 1993, he was with Hanjin Electronics Co. From Oct. 1993 to Feb. 1999, he was with the CDMA R\&D center of Samsung Electronics Co. Since Mar. 1999, he has been with the School of Electrical, Electronics and Communication Engineering,Korea University of Technology and Education, where he is currently a professor.His research interests are in the areas of wireless communications, mobile communication, and wireless sensor networkswith particular emphasis on wireless localization. 
International Journal of Control and Automation Vol.7, No.5 (2014) 